\title{
Autonomous Image to Model Registration
}

\author{
Alan I Chao \\ ALPHATECH, Inc. \\ Burlington, MA, USA \\ alan.chao@alphatech.com
}

\author{
Doug R. Morgan \\ Morgan Consulting \\ Los Altos, CA, U.S.A. \\ dougrm@sprynet.com
}

\begin{abstract}
Current production of geospatial site models is a labor intensive process. Consequently, automated/semi-automated solutions are sought to improve the production timeline. Automated image to model registration algorithms enable the identification of regions for focused data revision and the association of information for model refinement. Image to model registration is a sensor collection parameter estimation problem to optimize the spatial correspondence of information between the image and the model. This paper describes an automated algorithm based on the association of edge features to extract tie-points for sensor parameter estimation. These features are generated from the model through a predicted image of the site using the sensor model and available collection geometry. A key feature of this algorithm is the explicit incorporation of uncertainty in the registration process. Sample results using IKONOS satellite imagery are provided.
\end{abstract}

Keywords: Registration, site models, edge matching, bundle adjustment, uncertainty propagation.

\section{Introduction}

Image to model registration is an image collection (sensor) parameter estimation problem where the objective is to find the optimum spatial correspondence of information between the image and the model. A general approach to this problem is to extract features from the image and the model, which constitutes a representation of the information contained in the data, and use these features to establish spatial correspondence. In this context, the registration problem can be decomposed into two coupled sub-problems:

- Feature Selection and Association: This is the problem of extracting salient features across the image and the model, computing the optimal correspondence between these two sets of features, and scoring this correspondence or match for a given set of sensor parameters that acquired the image.

- Sensor Parameter Estimation: This is the problem of estimating the sensor parameters given feature correspondences or tie-points across the image and the model. In photogrammetry this is referred to as bundle adjustment.
The coupling between these two problems arises from the fact that the optimal correspondence computed in the feature association problem is necessary to solve the parameter estimation problem; while at the same time, the optimal sensor parameters are necessary to solve the feature association problem. The challenge to automating the registration process is the robust extraction of salient features between the image and the model and the robust association of these features to generate feature correspondences for the bundle adjustment. This paper describes an automated registration algorithm that combines state of the art feature extraction and matching technology from the computer vision discipline with rigorous photogrammetric bundle adjustment. This algorithm directly addresses the coupled sub-problems and the associated challenge.

\section{Registration Algorithm}

The process flow for the image to model registration algorithm is presented in Figure 1. The inputs are the collected image and its metadata, which contains the initial sensor collection parameters, and the site model to which the image is to be registered. Also input is a model of the sensor collection process, which is referred to as the sensor model.

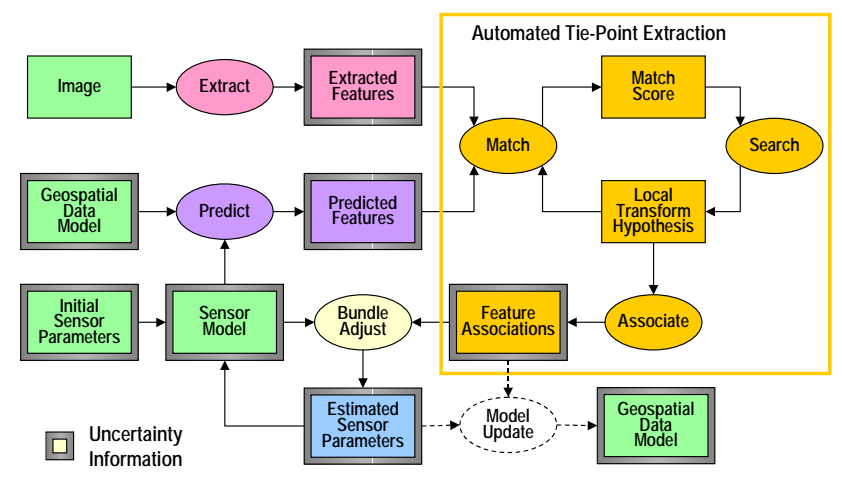

Figure 1. Image to Model Registration Process Flow.

Within this process, the features are extracted in the image domain where a corresponding image is predicted from the model under the constraints of the sensor model. The goal here is to predict an image that corresponds to the collected image in accordance to the model. Errors in this prediction are due to errors in the sensor model (i.e., position errors for the sensor) that are to be corrected. Given the two sets of extracted features (i.e., one from the 
collected image and one from the predicted image), the objective is to generate an association across these two feature sets to establish tie-points. This is performed in the local match-search iteration loop where local transformation hypotheses are evaluated to establish local correspondences for subsets of the features. Each local correspondence or feature association then result in a tiepoint measurement that goes to estimate the sensor parameters in the bundle adjustment. The bundle adjustment process accounts for uncertainties in the feature extraction and matching process as well as prior uncertainties in the imaging sensor and model. The estimated sensor parameters as well as their estimated posterior uncertainties are the outputs of the registration process, and there can be an additional iteration loop to minimize residual errors.

\subsection{Site Prediction}

Site prediction is performed by the Scenesim package. Scenesim projects geometric shapes into an image plane and accounts for illumination, visibility, and viewing geometries. Scenesim uses industry standard OpenGL functions to carry out the geometric rendering of a scene model composed of triangular facets. The output of Scenesim is an image of the scene with the shadowing and projection geometry for the particular sensor and collection.

The pixel values of the predicted image contain numerical IDs corresponding to the label on the triangular facet that is visible to the sensor at the corresponding pixel coordinate. Pixels corresponding to facets that are visible but not illuminated are assigned the ID corresponding to shadow. The numerical IDs allow for trace back from the rendering of the scene model to the 3$\mathrm{D}$ objects of the scene. The user can choose the ID to suit his purposes, such as naming the specific facet, the object to which the facet belongs, or the type of object to which the facet belongs. The rendered image can be fed to feature extraction algorithms and the resulting features directly matched against features extracted from a real image.

Figure 2 illustrates how Scenesim renders a scene. The steps are:

1. Determine illumination. An illumination depth buffer is computed of the scene from the point of view of the illuminator. For optical sensors, this is usually the Sun direction relative to the scene. To reduce sampling artifacts in the final result, the sample spacing is chosen to be less than the image sample spacing. Each cell of the illumination depth buffer is filled with the numerical ID of the illuminated facet that projects into the cell and is nearest the illuminator.

2. Determine visibility. A visibility depth buffer is also computed by depth buffering the scene from the point of view of the sensor. The visibility depth buffer is created with sampling matching that of the final image to be predicted.

3. Project model. Model points that are illuminated and visible are projected into the image plane, forming the predicted image of facet IDs. The predicted image is initialized to shadow ID. Then the angle-angledepth information of each cell in the illumination depth buffer is converted to a 3-D point that may be painted, depending on visibility, into the predicted image with the cell's ID value. For an optical sensor, only those cells that map closer to the sensor than the visibility depth buffer are drawn into the predicted image. The last cell mapped to an output pixel gives that pixel its ID value.

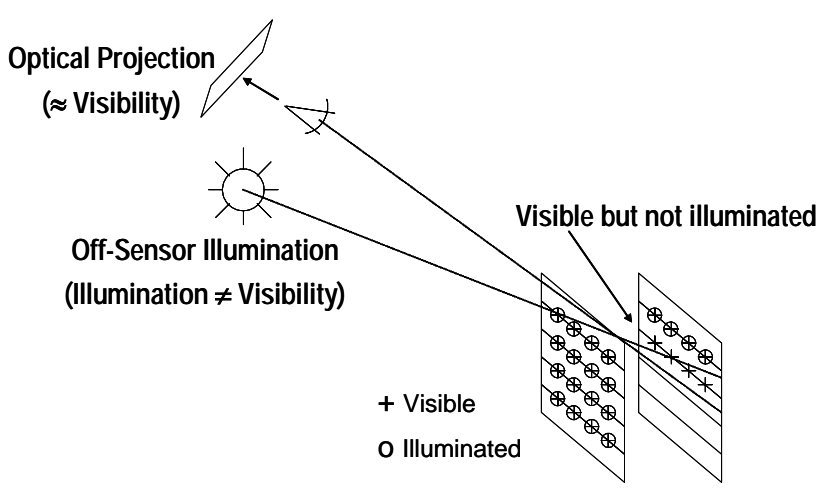

Figure 2. Overview of Scenesim Rendering.

\subsection{Automated Tie-Point Generation}

The automatic generation of tie-points in the registration process involves associating features extracted from the collected and predicted images. The challenge is in identifying and extracting salient features across the data sets and to robustly match these features. In the present algorithm, edge features are used. For images, edge features are image pixels where there is a local maximum in the image gradient in the direction of the gradient. Edges are important topological features for registration since they correspond to boundaries between regions in the image with contrasting intensities. In addition, edges are very accurate identifiers of these boundaries in terms of spatial localization. Consequently, they are often used as features for image registration [1]. A standard Canny algorithm [2] is used for edge extraction. The Canny edge detector uses an approximation to an optimal filter for detecting step edges corrupted by Gaussian white noise.

Once the features are extracted from the collected image and the predicted image (from the model), automated tiepoint generation seeks to find the local transformation that maps one set of features to the other that results in the best spatial correspondence between the two sets. This is an optimization problem. The resulting optimal local transformation provides a tie-point measurement in that it associates a set of features in the image to a set of features in the model. To formalize the optimization objective, a 
match metric is needed that scores the spatial correspondence between the two sets of features.

In the present algorithm, distance transforms are used to score the match correspondence. Distance transforms are measures used to denote the distance between two sets of points. In this context, the two sets of points are two sets of feature points, one extracted from the collected image and one extracted from the predicted image. The distance transform is used to determine the distance or the degree of match between these two sets of features. One example of a distance transform is the directed Hausdorff distance from set $A$ to set $B$ [3],[4], which is defined as

$$
h(A, B)=\max _{a \in A} \min _{b \in B}\|a-b\|
$$

In the context of matching, the directed Hausdorff distance quantifies the worst case deviation in matching the points in $A$ to the points in $B$ since it is the distance to its nearest neighbor in $B$ for the most mismatched point in $A$. That is, the directed Hausdorff distance places an upper bound on the mismatch distance when matching $A$ to $B$. The implicit feature mapping assumed by this metric is a many-to-many correspondence. That is, many points in $A$ may be close to the same point in $B$. For matching topological features, this assumption is consistent since no one-to-one correspondence should be assumed between the feature sets. In the context of registration, the transformation (that maps the points in $A$ to match the points in $B$ ) that minimizes such a distance is sought, thereby minimizing the worst-case match distance.

The Hausdorff distance can be readily extended to denote the best partial distance between two sets. This is useful when there are certain points in the set $A$ that are extra features and do not have a true corresponding point in $B$. In this context, the partial directed Hausdorff distance is defined as

$$
h_{K}(A, B)=K_{a \in A}^{t h} \min _{b \in B}\|a-b\|
$$

where the $K$ th ranked distance is chosen rather than the largest. This definition has a nice interpretation in that the metric chooses the $K$ best matching points in $A$ to quantify the match distance.

Although the partial Hausdorff metric is robust to extra and missing features in either feature set, properly setting the parameter $K$ is difficult. This is because $K$ is data dependent. To get around this issue, a better distance metric, motivated by the above Hausdorff metrics, is

$$
d(A, B)=\frac{1}{N_{A}} \sum_{a \in A} \min \left(\min _{b \in B}\|a-b\|_{\Sigma}, d_{\max }\right)
$$

This is known as the average statistical distance. Instead of returning the worst-case match distance, this metric returns the average match distance. To avoid the problem that missing and extra features can bias this average distance, a threshold is placed on the best match distance for each feature point. If this best match distance is greater than the threshold, then it is assumed that that feature is an extra feature with no corresponding match in the other feature set (or the corresponding feature is missing). Consequently, this large mismatch distance does not enter into the average distance. The threshold is interpreted as a noise floor for mismatches. Given that the distance measure is a Mahalanobis distance with the association covariance $\Sigma$, this threshold also has the interpretation of a $X^{2}$ confidence measure.

The above metrics only quantifies the mismatch distance from $A$ to $B$. For registration, minimizing the mismatch from $B$ to $A$ is also desired. This motivates the definition of a bi-directional distance metric

$$
d_{\mathrm{BI}}(A, B)=\max (d(A, B), d(B, A))
$$

This distance is simply the maximum between $d(A, B)$ and $d(B, A)$. Intuitively, if this distance is $D$, then the worst case mismatch distance between the best matching points in both sets is $D$.

Finally, the process of identifying tie-points requires searching for the local transformation resulting in the best match between the two sets of features as quantified by the match metric. In the present algorithm, the local transformation space is local translation of one feature set relative to the other. A hierarchical grid search algorithm over the 2-D translation space is used.

This hierarchical approach starts with a coarse discretization over the search range and proceeds recursively to search over finer grids in only those areas where improvement in the current best score is likely. Clearly, a finer grid search in the vicinity of the current best score needs to be performed. However, the current best score can be in the vicinity of a local critical point and not the global optimum. One solution is to keep all current points whose score are within $\delta$ of the current best score, where $\delta$ is an algorithmic parameter, and to continue to the next finer level of search only around those points. This approach is summarized in the following steps for the case in which the transformation space is the 2-D translation space:

1. Begin with a coarse grid using an initial grid spacing of $S=S_{0}$ in each dimension. Evaluate the match score over all points in this grid and find the best current score.

2. Go through the list of grid points. For every point whose score is within $\delta$ of the current best score, evaluate the match function over the range [-S/2, $S / 2]$ x $[-S / 2, S / 2]$ at a grid spacing of $S=S / 2$. Add these new points to the list and discard all points whose score is not within $\delta$ of the current best score from the list. Find the new current best score.

3. Repeat step 2 until the grid spacing $S$ is equal to the desired search resolution.

This approach is, in general, sub-optimal in that there is no guarantee that the result will be the best transformation given the desired search resolution. Optimality can be shown for the case in which the match score surface is 
Lipschitz, and the Lipschitz constant is known. This is the case for the directed Hausdorff distance under translation poses where the Lipschitz constant is unity [5],[6]. In this case, the value of $\delta$ (which will now be a function of the current grid spacing) can be set in accordance to the Lipschitz constant so that all points whose match score cannot possibly increase to be above the current best score in its vicinity can be eliminated from the list. However, although the match surface for either the partial bidirectional Hausdorff distance or the average statistical distance match metric is Lipschitz, the Lipschitz constant is not known a priori for these cases and depends on the feature data to be matched.

Furthermore, the parameters $S_{0}$ and $\delta$ can be used to trade between optimality and efficiency. Clearly, by using a fine initial grid, the chances of finding the optimal point are improved, but at a cost of increasing computational requirements for the search. Likewise, if $\delta$ is increased, then the requirement for rejecting search points becomes stricter. This forces more points to be kept in the next finer level of search. As a result, the chance of finding the optimal point is increased but again at an increased cost. Intuitively, $S_{0}$ should be set to half the estimated convergence range for the global optimum. Doing so will insure that one of the initial coarse grid points is close to the global optimum. In the current implementation, $S_{0}$ is set so that there is at least four initial coarse grid points over any one dimension of the search range. Also, $\delta$ should be set initially to $\eta S_{0} / 2$ where $\eta$ is the estimated Lipschitz constant for the match surface. In addition, $\delta$ should be reduced by a factor of two for each level of the search as the grid spacing is halved.

\subsection{Bundle Adjustment}

Bundle adjustment is used to estimate registration parameters and to compute error covariances. All bundle adjustment is based on a Bayesian network model of the random variables (RVs) in the scenario of sensors, maps, world objects, and features. Bayesian operations on the network compute prior and posterior probability distributions of the various RVs of the network. Posterior distributions are conditioned on observed features, prior distributions are not. Different prior and posterior distributions may describe many items of interest, including:

- Uncertainty of sensor position and attitude.

- Uncertainty of an image feature location projected to a map or to the ground.

- Uncertainty of a map feature location projected to the ground.

- Uncertainty of a world object location with respect to a map or to the ground.

- Components of prior uncertainty of an image feature location on the ground to causes such as sensor positions and attitude, map error, object error with respect to a map, etc.
Bundle adjustment is based on a Gaussian model for small offsets of system parameters and observations away from nominal values. The Gaussian model is typically constructed from many linear Gaussian relations that describe sets of offsets as being related by linear transformations plus independent Gaussian noise. The collection of such relationships is a Bayesian network, as illustrated in Figure 3. In a Bayesian network, each node of the graph denotes a conditional probability distribution of the random variables in the node conditioned by the variables in nodes on incoming edges. Any subset of variables may be constrained to observed values. The entire graph represents the product of the various conditional density functions.

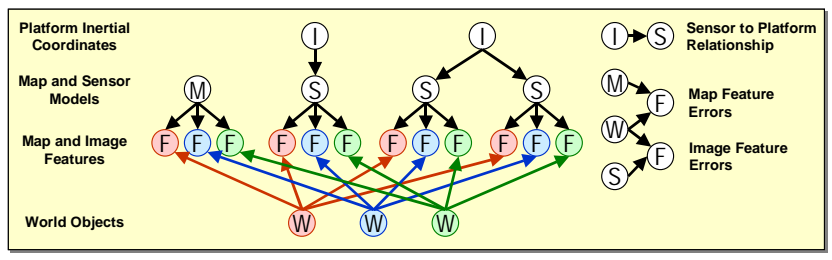

Figure 3. Bayesian Network for a Photogrammetric Scenario.

Figure 3 shows the causal relationships between sensor platforms, sensors, world objects, image features, map makers (treated as another kind of sensor), and map features. The sensor platform inertial position and orientation histories partially determine the attached sensor exterior orientation histories. A platform may have multiple sensors attached. Each sensor and map maker sees objects in the world and subsequently produces image and map features with particular locations and attributes. For small perturbations (and before values are observed), each of these relationships is approximated by a linear Gaussian relationship: $y=y_{m}+H x+v$ where $y$, $x$, and $v$ are Gaussian vectors with $x$ and $v$ independent and $v$ having zero mean, $H$ is a compatibly dimensioned matrix (of partial derivatives of a more accurate nonlinear relationship), and $y_{m}$ is the mean of $y$ when $x$ is given to be zero. Each node of the Bayesian network represents one such equation, with the variables in the node playing the part of $y$ and the collection of variables in nodes on incomings edges playing the part of $x$. The parts of $y_{m}, H$, and $v$ are parameters of each node, but are not shown. In a Bayesian network, any variable is conditionally independent of any ancestor variable given all its parent variables.

The general solution for any such collection of distributions is to create one large Gaussian distribution containing all the variables and then to compute the marginal distributions of interest (possibly after applying constraints due to observations). For $N$ variables, this takes on the order of $N^{3}$ operations. With today's computing hardware, problems with 1000 variables can be solved in a few seconds with the general method. Exploiting the many independencies in the causal relationships of the Bayesian network allows problems 
with many thousands to millions of variables to be handled.

To take advantage of the independencies, the initial non-tree-shaped network of the scenario is first converted an equivalent tree form that supports particularly simple solution strategies. This conversion combines all platform, sensor, and map offsets into one node and for each world object combines all features associated with that object into a single node. Combining involves multiplying conditional distributions.

The solution strategy for finding the prior or posterior distribution of a subset of variables of interest proceeds as follows:

1. For each observed RV, replace the unobserved distributions involving that RV with observed ones and constrain each observed RV to its observed value.

2. For each leaf node or node containing the unique occurrence of some RV or RVs, marginalize away unwanted variables.

3. If unwanted variables remain, multiply distributions such that unwanted variables appear in leaf nodes and iterate with step 2.

4. If necessary, multiply and/or conditionalize the final nodes containing exactly the desired variables to obtain the desired proper probability distribution that integrates to 1.0 .

The final result is a new Bayesian network consisting of a single node. The node's RVs are exactly the set of desired variables and its distribution is the desired prior or posterior distribution.

The Bayesian network exploits the same bandedbordered covariance matrix structure often seen in photogrammetric bundle adjustment algorithms. The presentation of the Bayesian network algorithm differs, but the computational possibilities are the same. The banded-bordered structure can best be seen in a covariance matrix of all variables that has been ordered so that all sensor and map variables come first, then world object parameters, and finally image and map features. The covariance is block diagonal except that the rows and columns corresponding to features have borders of nonzero correlations with sensor parameters and bands of nonzero correlations with the world object parameters. This reflects the tree structure with every feature cluster connected to the sensor variables (border) and with each feature cluster connected to a single world feature (band).

Unlike the more common algorithms used in photogrammetry, the system developed under this effort uses orthogonal triangulation (the QR decomposition algorithm) to manipulate inverse square roots of covariance matrices. The computations are similar to those found in the information matrix form of the square root Kalman filter. This approach has the advantages of better numerical conditioning than non-square-root algorithms and of computing covariance matrices that are always positive definite. No numerical anomalies were seen using this algorithm even though there was no attempt to balance or condition the range of different RVs (e.g., the tightly varying pointing angles in radians for sensors in orbit are freely mixed with the much more widely varying feature location in meters or pixels).

\section{Results}

\subsection{Data}

The registration algorithm is demonstrated using commercial IKONOS panchromatic imagery with a spatial resolution of one meter. The imaged site is a small town center consisting of a collection of buildings of varying size and shape in an area roughly 400 by 400 meters. The site model is constructed from digital elevation data at one meter postings. Facetization of the elevation data is performed using a standard triangulation algorithm [7]. The IKONOS image and the site model are shown in Figure 4 and Figure 5, respectively.

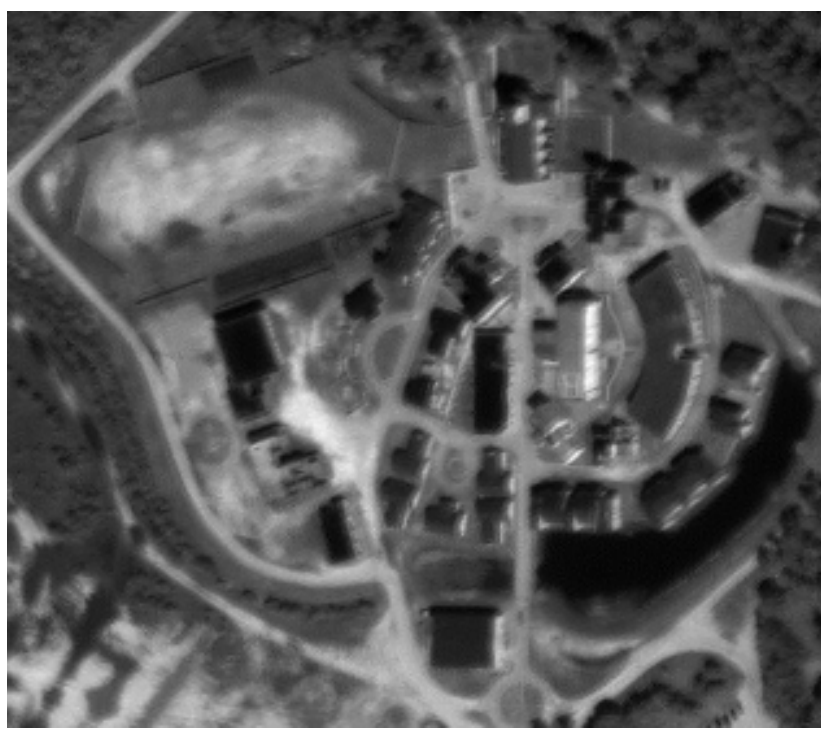

Figure 4. IKONOS One Meter Panchromatic Image.

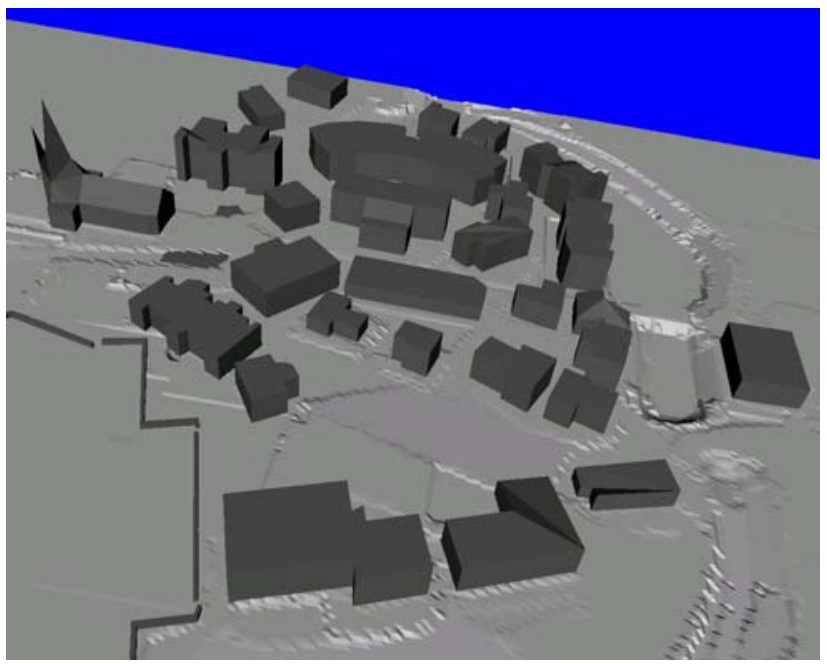

Figure 5. Facet Site Model. 


\subsection{Registration Results}

Using the sensor collection parameters in the image metadata, a predicted image of the site is generated by Scenesim, as shown in Figure 6. In Figure 6, the spatial errors of the site model, which is set at $\sigma_{x}$ and $\sigma_{y}$ at 0.5 meters and $\sigma_{z}$ at 2.0 meters, is projected into the predicted image and shown as the green ellipses (3- $\sigma$ uncertainty) for a few chosen points in the site.

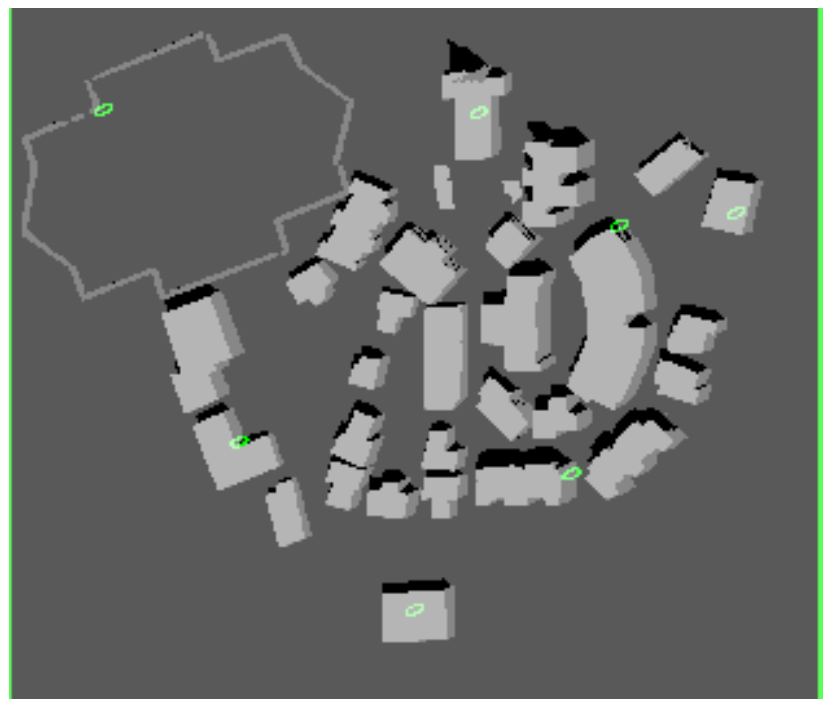

Figure 6. Predicted Image Corresponding to IKONOS Image with Projected Site Model Errors.

Edge features are extracted from both the predicted and collected images. In the predicted images, the total spatial uncertainty of the edge feature is composed of both the site model uncertainty and the feature extraction uncertainty. In the collected image, the feature spatial uncertainty contains only the extraction uncertainty component. For both predicted and collected images, the extraction uncertainty is set with $\sigma$ at one pixel.

An initial overlay of the predicted edge features on the collected image (in the collected image plane) is shown in Figure 7 where the green predicted features are overlaid on the white collected features. This overlay quantifies the initial registration error. The white ellipses in Figure 7 denote the projected sensor error ellipses $(3-\sigma)$ in the image plane.

Figure 7 shows that the initial error can be quite large (i.e., same order of magnitude as the overall size of the scene) and is dominated by translation. Consequently, the algorithm first attempts to correct for this gross translational error globally. It does so by considering all of the extracted features in both the predicted and collected images and seeks to find the optimal translational transformation that maximizes the match. The result of this global match process is given in Figure 8 . Here the corrected predicted features are shown in cyan overlaid on the white collected image features. This result shows that the gross translational error has been corrected by the global match process.

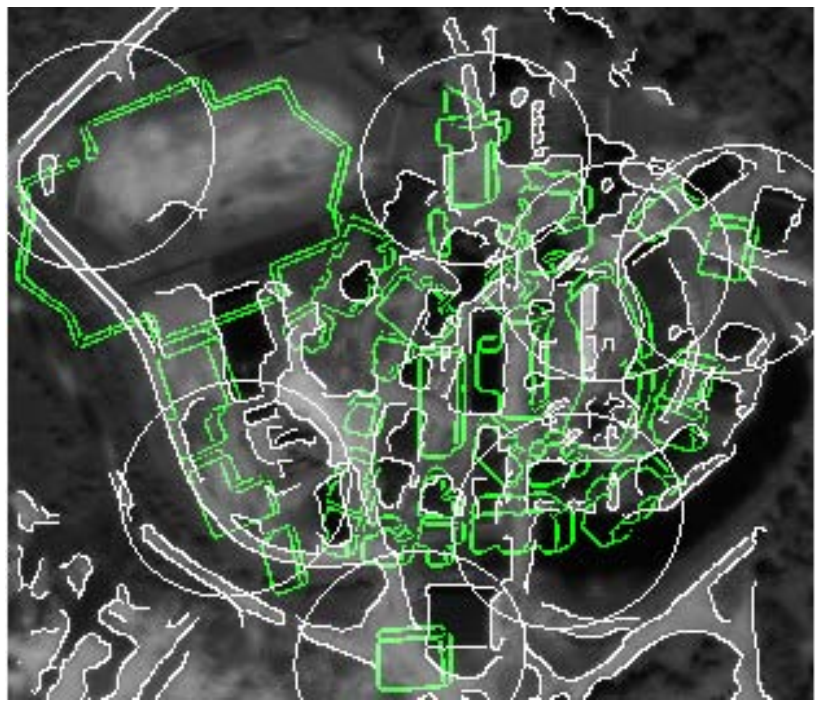

Figure 7. Initial Edge Overlay Showing Registration Error and Projected Prior Sensor Error Covariance

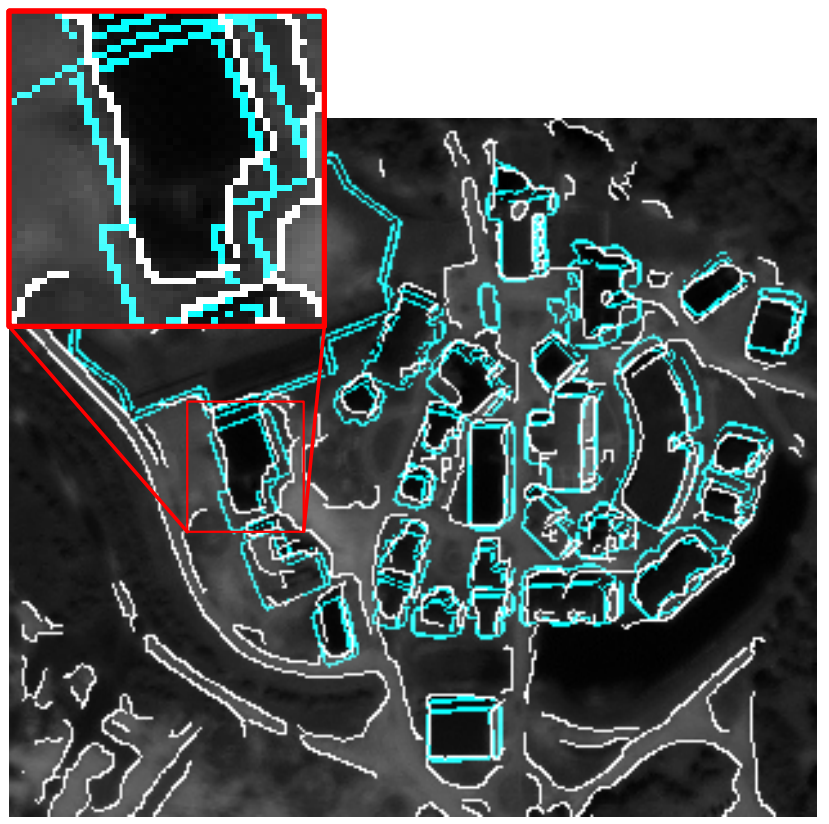

Figure 8. Edge Overlay after Global Translation Correction.

The global translational correction, however, does not completely register the image to the model. Looking at the zoomed-in inset in Figure 8, there are still residual registration errors that do not fit the translational error model. The purpose of the global translational correction is to get close to the registration solution. The algorithm then proceeds to perform local feature matching over a set of independent regions spatially distributed across the image. These regions are currently picked a priori by hand. The criteria for picking these regions are:

1. They need to be distributed over the image. Each region will contribute a tie-point measurement to bundle adjustment. Distribution across the image will lead to a better-conditioned solution. 
2. They need to be disjoint. This is to insure independence in the tie-point measurements.

In Figure 9, the four regions picked for this case are shown by the cyan circles. These correspond to significant buildings or groups of buildings in the site.

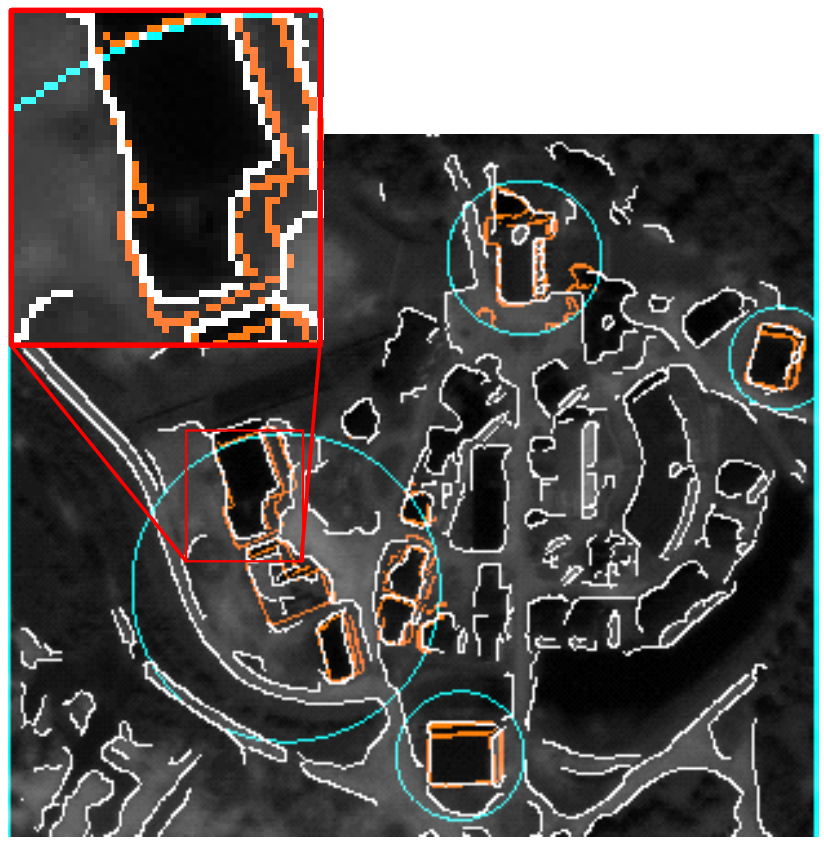

Figure 9. Matching Local Edges for each Independent Region.

For each region, the local match process is identical to the global match process except that only the features contained in the region are considered. Specifically, the local match process seeks to find the local translational transformation that maximizes the local match for each region. The results from these local matches are shown in Figure 9. Here, the tan features correspond to the locally corrected predicted features for each region.

Combining the global and local match solutions for each region generates the tie-point solution for that region. This is visualized in Figure 10. Here, the green circle denotes the location of each region initially (i.e., prior to global matching). After global matching, the region is shifted to the cyan circles, and after local matching the region is shifted again to the tan circles. Choosing any point in each region (i.e., choosing the center of the circles) and tracing its location after global and local matching produces a translation vector for that point. In other words, the end-points of these vectors (one for each region) form a pair of tie-points since the local matching process determined that the two points are associated. These tie-point measurements are independent since the features in the disjoint regions are independent. These tie-point measurements are subsequently used to adjust the sensor parameters. After bundle adjustment, a new predicted image for the site model is generated based on the adjusted sensor parameters. This is shown in Figure 11 where the blue features correspond to the edges extracted from the new predicted image overlaid on the collected image. This result show that the registration solution is quite good after one bundle adjustment iteration and the algorithm terminates. The red ellipses in Figure 11 are the 3- $\sigma$ spatial error covariances after registration, which includes the projected posterior sensor parameter errors, the projected modeling error, and the edge extraction error.

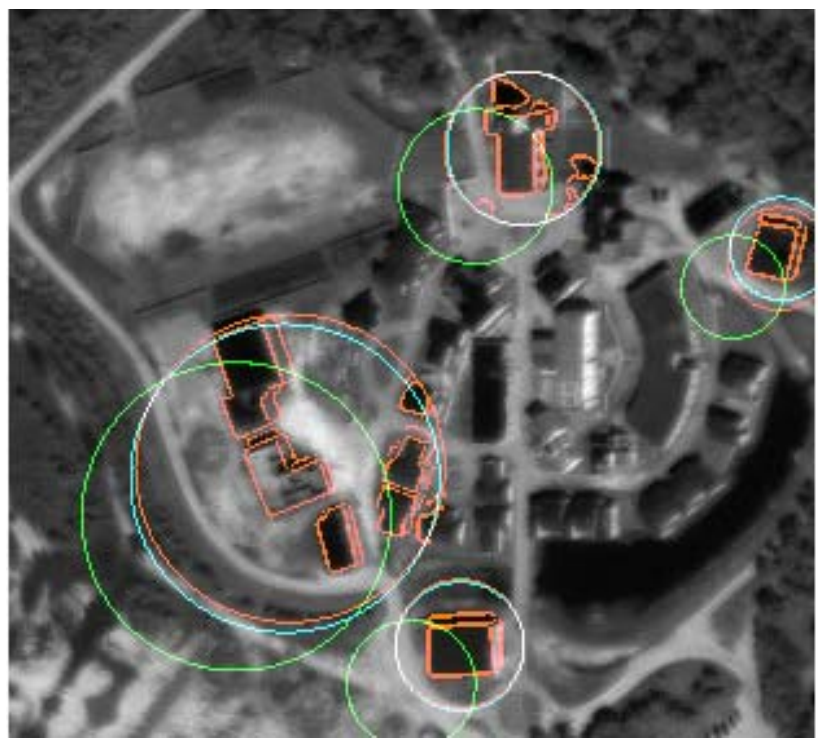

Figure 10. Tie-Point Measurement for each Independent Region

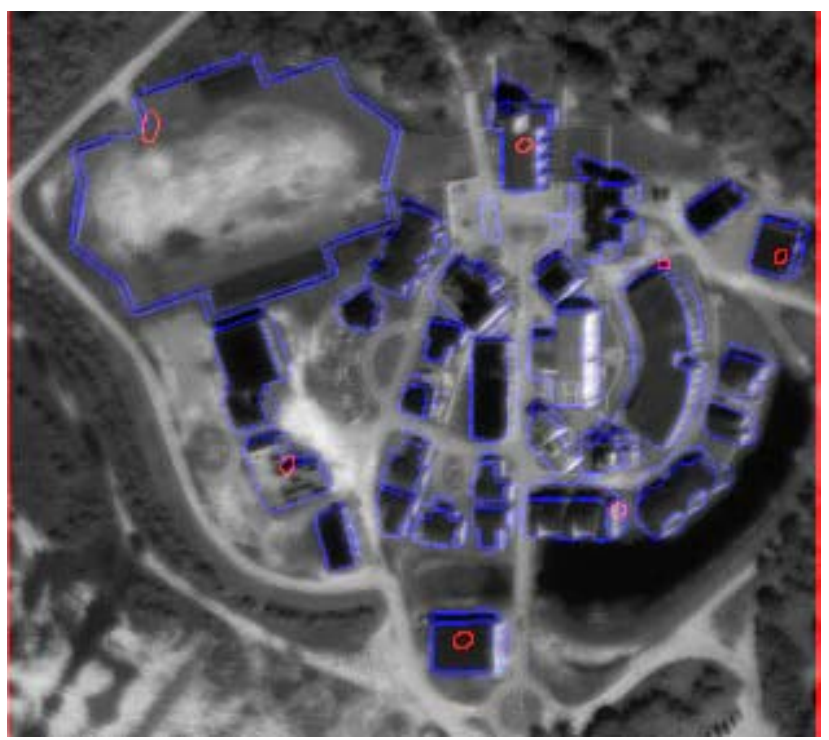

Figure 11. Bundle Adjusted Registration Solution with Posterior Covariance.

As shown in Figure 11, the resulting registration accuracy in terms of a 3- $\sigma$ posterior error ellipse is on the order of 3 resolution cells in the areas that supplied a measurement for bundle adjustment. This translates to roughly a 3 meter error relative to the site model. This is on the order of the site model errors. Consequently, the 
resulting registration solution can be used to detect significant change of the scene relative to the postulated site model. Computationally, the processing time for a $\mathrm{C}++$ implementation of the algorithm on a 512 by 512 pixel IKONOS sub-image covering the site is under 20 seconds on a $1 \mathrm{GHz}$ Pentium III computer running the Linux operating system. Using the registration solution, the image is textured mapped to the rendered site model as shown in Figure 12.

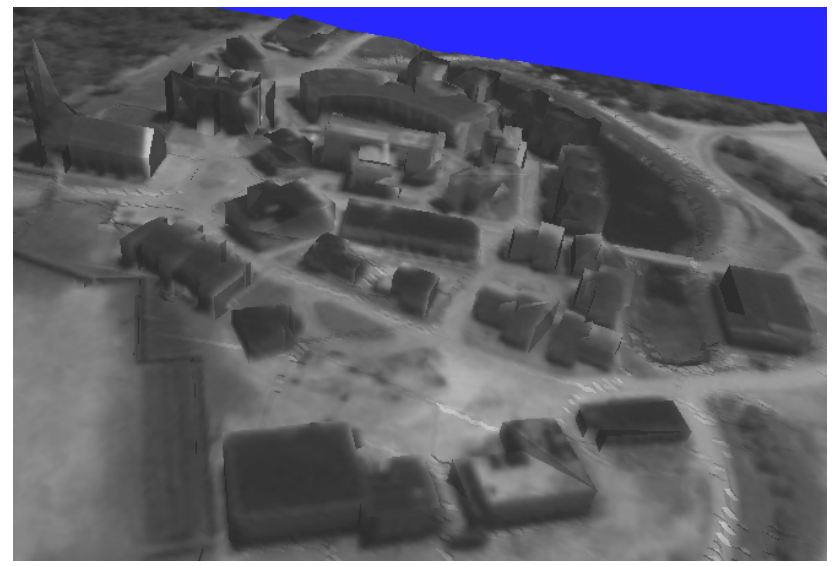

Figure 12. Registered IKONOS Image Texture Mapped to Site Model.

\section{Conclusions}

This paper presented an autonomous image to model registration algorithm. This algorithm provides the following technology advances:

- Automated generation of ground control matching patterns from models in the form of a predicted image.

- Adjustable model for push-broom sensors such as IKONOS.

- Matching of edge features to automatically identify tie-point candidates.

- Bundle adjustment of sensor parameters incorporating local feature matches as measurements.

- Explicit incorporation of uncertainty in the registration process. The resulting registration solution provides posterior error covariance estimates to quantify uncertainty for downstream change detection and model update.

On sample IKONOS imagery over a typical site, this algorithm produced accurate registration solutions that are of the order of the site modeling errors, which is sufficient for use in subsequent change detection.

\section{References}

[1] Kwok, R., E. Rignot, J. C. Curlander, and S. Pang, "Multisensor Image Registration: A Progress Report," JPL Tech. Doc. D-6697, Jet Propulsion Laboratory, Pasadena, CA., 1989.
[2] Canny, J. F., "Finding Edges and Lines in Images," MIT Tech. Report, AI-TR-720, Artificial Intelligence Laboratory, MIT, Cambridge, MA., 1983.

[3] Huttenlocher, D. P., G. A. Klanderman, and W. J. Rucklidge. "Comparing Images Using the Hausdorff Distance," in IEEE Trans. on Pattern Anal. and Mach. Intel., Vol. 15, No. 9, pp. 850-863, September, 1993.

[4] Alt, H., B. Behrends, and J. Blomer, "Measuring the Resemblance of Polygonal Shapes," in Proc. Seventh ACM Symp. Comput. Geometry, 1991.

[5] Ryan, T. W., "MSTAR Indexing Module (IXS)," WL-TR-97-1018, Interim Report for Period: 06/01/9512/01/96.

[6] Irving, W. W. and J. K. Johnson, "SAR/FLIR Sensor Fusion for ATR with 3-D Model-Based Reasoning," ALPHATECH Technical Report TR-868, contract F33615-97-C-1107, Burlington, MA., February 1998.

[7] Narkhede, A., and D. Manocha, " Fast Polygon Triangulation Based on Seidel's Algorithm," Paeth, Alan W., ed., Graphics Gem V, AP Professional, Boston, pp. 394-397, 1995. 